\title{
Annotation
}

\section{Congenital cataract, a cause of preventable child blindness}

Congenital cataract is numerically the most important cause of remediable blindness in children, being far more common than, for example, retinoblastoma or congenital glaucoma.

The management of congenital cataract has been profoundly altered during the last $\mathbf{1 5}$ years as the result of a number of developments. Since the work of Wiesel and Hubel, ${ }^{1}$ and that of many others, clinical ophthalmologists have been aware of the relevance of amblyopia in the management of visual disorders in childhood. Amblyopia implies a loss of vision that occurs as a result of visual deprivation during a sensitive period of development of the visual system. The neurophysiological and anatomical changes that are the substrate of the visual loss occur throughout the visual pathway possibly even from the retinal receptors to the cortical areas where perceptual processing occurs. From human analogies of the experimental animal it has been concluded ${ }^{2} 3$ that in infancy there may be a period of grace of up to age 2 months when the effects of visual deprivation are of less consequence in that they are less profound and reversible. After this period there are 6 to 18 months of increased sensitivity, the human equivalent of the experimental animal's critical period, followed by a gradually declining sensitivity for several years so that by age 9 or 10 years a child's visual system is not deranged by degraded visual imput. Amblyopia can be caused not only by opacity in the optical media of the eye-such as cataract-but also by a blurred retinal image such as occurs in uncorrected aphakia (the absence of the lens from its normal site, for any reason, with the loss of its refractive role). The impact of greater understanding of the mechanisms of amblyopia on the management of cataract has been twofold: surgeons now realise that most children with congenital cataract who require surgery in infancy should have that surgery completed by age 2 months; and early accurate correction of aphakia is essential if a sharp retinal image is to be produced during the sensitive period.

There have been important changes to the surgery of congenital cataracts in recent years ${ }^{45}$ and these have substantially improved the short- and long-term prognosis for the eye. The eyes of very young infants can be operated on with safety, the likelihood of serious complications-such as retinal detachment or glaucoma-is reduced, fewer operations are required, and rubella cataracts can be treated surgically in the same way as other congenital cataracts.

Advances in contact lens technology have made it possible to use extended wear contact lenses for the correction of aphakia in infants. Contact lenses are readily fitted without anaesthetic and the tolerance rate is high despite the high refractive error. It is possible in most cases to overcorrect the aphakic eye to make the lens eye combination myopic so that the infant can clearly see objects at some point within 1 metre which is his main sphere of visual interest in the first months of life when the brain is particularly sensitive to the degraded visual input. Satisfactory correction of aphakia cannot be achieved in infants by means of spectacles which, because of the very high power lenses required, are impracticably heavy and optically unsatisfactory. The use of intraocular lens implants in infants is not recommended at the present time because of uncertainty about long-term complications.

\section{Presentation}

Unilateral congenital cataracts present because of the appearance of a white pupil, small eye, or unilateral squint noticed by parents or relatives. The smallness of the eye is usually marginal and it may be thought that the other eye is large. The white pupil is more often described as milky, hazy, or as 'just not looking right', it may be seen only in certain lighting conditions and should therefore be a symptom to be believed even if not seen.

Bilateral congenital cataract often occurs if other members of a family are affected, and it is these patients who are best poised for ideal management. Parents notice early that their baby does not seem to look at them and they may also notice white pupils. By age 3 months a baby with severe bilateral cataracts will start to develop nystagmoid movements on attempted fixation and this, or an associated squint, is generally the mode of presentation in what are now regarded as late cases, since they seem to indicate that the visual system is already reacting to visual deprivation. 


\section{Diagnosis}

Diagnosis at the clinical level is generally made initially by the paediatrician who listens to the mother's worries about the baby's vision, the appearance of the pupils, or the presence of nystagmus or squint. Careful inspection of the eyes may show the small eye or the white pupil, but the absence of a white pupil does not exclude a cataract since the opacity may be posterior in the lens. One useful technique is to look at the eye with an ophthalmoscope as though one is going to look at the fundus but keeping about a foot away; the absence of a red reflex is a clue to an opacity in the refractive error. Closer inspection with the ophthalmoscope is possible if the lens system of the ophthalmoscope is set at +20 dioptres. If a cataract is suspected, referral to an ophthalmologist should be arranged as an urgency, especially if the baby is less than 1-year old.

A search for the aetiology of the cataract is important but should not delay the management of the cataract itself.

About one-third of congenital cataracts are genetically determined, and there are also certain morphological types that are found more frequently in genetically-determined cataracts. However, some genetically-determined systemic diseases are also associated with cataract - for instance galactosaemia, Lowe's syndrome, and lactose intolerance as well as numerous syndromes of unknown causes of which the most common are the HallermannStreiff, Rubinstein-Taybi, and Conradi-Hünermann syndromes.

Cataracts are often found in babies with Down's syndrome, and also with trisomy 13 and 18 , and in Turner's syndrome. In the congenital rubella syndrome the cataracts are generally present at birth and about one-third are uniocular. ${ }^{6}$ They are usually present as a part of the whole syndrome but occasionally the cataract is the only abnormality detected initially. The early detection of the cataract and management of it are especially important in view of the high incidence of deafness as the child grows older. Cataracts are less common in other prenatal infections. Metabolic causes include galactokinase and transferase deficiency, Lowe's syndrome, and hypocalcaemia.

Investigations should be carried out after consultation between ophthalmologist and paediatrician, each of whom will be able to exclude certain causes on clinical grounds thus saving time and money on unnecessary tests. This particularly applies to galactose metabolism tests as these are expensive to carry out in detail but inexpensive to screen for.

\section{Management}

It may be difficult to decide whether a cataract requires treatment. The optical disadvantages of aphakia, combined with the risks and possible failure of treatment, make it inadvisable to operate and start optical treatment unless it is certain that the child will be visually handicapped unless he is treated. Most cases fall clearly into the 'obviously blind' or 'obviously sighted' category but the management of border-line cases must be decided by a variety of techniques. If some form of infant vision testing device-such as visually-evoked cortical responses or forced preferential lookingare available the choice may be easier. However, these techniques are not widely available nor are they universally accepted as an accurate index of vision in infancy. The ophthalmologist may make some judgement by the clarity with which he sees the fundus with an ophthalmoscope, but this technique must be inaccurate because of the very poor correlation between the degree and site of the opacity in the refractive media and the vision of the child in cases where this can be measured. Therefore the doctors managing the child must rely on behavioural observations in borderline cases. If the infant fixes objects steadily without nystagmus or squinting, and responds to subtle clues-such as a silent smile or movements of small objects-it is unlikely that his vision is sufficiently poor to warrant early treatment. If there is any doubt there is very little to lose by reassessing the child after an interval of one or two weeks. In borderline cases, repeated examinations may help to make this critical decision.

\section{Bilateral congenital cataracts}

Bilateral congenital cataract is the most common cause of potentially treatable blindness in infancy. It now seems likely that, although the results are not universally successful, early surgery leads to better visual results provided that optical correction is started early. ${ }^{7}$ If diagnosed in the neonatal period it is reasonable to wait for 2 or 3 weeks before admitting the child to hospital for eye surgery in order to make the surgery somewhat easier. Unfortunately however, delays in diagnosis and referral result in most babies with bilateral severe congenital cataracts being seen by the ophthalmologist for the first time between 2 and 4 months of age. By age 4 months one must expect the effects of visual deprivation to be showing their mark and the counsel of perfection is that all patients should be referred early so that, if necessary, surgery and optical correction can be completed by age 2 months. After surgery the baby is fitted with continuous wear high gas-transfer contact lenses 
without anaesthetic. ${ }^{8}$ There is a substantial risk of corneal damage from these and frequent follow-up visits are necessary; this, together with a high rate of lens loss and rejection, must be explained to the parents at the beginning of the treatment. The contact lenses are not strictly necessary once the child is old enough to wear spectacles and we try to ensure that the patient is wearing spectacles all the time by age 3 and that he is wearing bifocals by school age.

The cost of contact lens treatment, both to the parents and to the NHS is admittedly high, perhaps between $£ 200$ and $£ 1200$ a year, depending on the numbers of lenses used and the distance from the contact lens department, but if one can show an improvement in the child's visual performance sufficient to shift it from one educational category to another, even the fiscal argument is clearly one-sided.

\section{Unilateral cataract}

Until recently it was universally agreed that the visual prognosis for eyes with unilateral cataract was so poor that surgery was indicated only for cosmetic reasons or to prevent glaucoma resulting from a 'hypermature' lens. Recently Beller et al. ${ }^{9}$ described a series of children who were operated and optically corrected within days of birth. This was followed by vigorous occlusion of the normal eye and careful monitoring of the vision of both eyes by psychophysical or electrophysiological means. By the time of publication 9 of their patients who had received ideal treatment had Snellen acuities of about 6/9. This series has yet to be reproduced at another centre but quite apart from its significance in the understanding of human amblyopia, it may be of some interest in the management of patients with unilateral cataract. This is especially important in view of the increased vulnerability to injury of a single normal eye when its fellow is amblyopic. ${ }^{10}$ The social relevance of extended, and possibly risky, treatment for a non-blinding condition is more a matter for debate than that of bilateral cataract.

\section{Rubella}

Rubella cataract still poses several problems. The notion that rubella cataract surgery was best delayed for some months or even years because of the risk of endophthalmitis has now been outdated by improved surgical technique, and bilateral rubella cataracts require early surgery like any others. In rubella there is probably a stronger than normal indication to treat unilateral cataracts since there is a substantial incidence of late visual deterioration due to macular degeneration. ${ }^{11}$

\section{Conclusion}

Studies in amblyopia have led to the wider recognition of the importance of early treatment of congenital cataracts. The management is difficult, especially for the parents and the child, but the enhanced visual result in the majority of cases justifies the difficulties and the risks. There is a clear responsibility on paediatricians and others concerned in the care of children to be aware of the importance of congenital cataracts and the need for prompt referral to the ophthalmologist.

\section{References}

1 Wiesel T N, Hubel D H. Effects of visual deprivation on morphology and physiology of cells in the cat's lateral geniculate body. J Neurophysiol 1963; 26: 978-93.

2 Vaegan, Taylor D. Critical period for deprivation amblyopia in children. Trans Ophthalmol Soc UK 1979; 99: 432-9.

${ }^{3}$ Awaya S. Stimulus vision deprivation amblyopia in humans. In: Reinecke R D, ed. Strabismus. Proceedings of the third meeting of the International Strabismological Association. New York: Grune \& Stratton, 1978: 31.

4 Rice N S C. Lens aspiration-a decade in retrospect. Trans Ophthalmol Soc UK 1977; 97: 48-51.

5 Taylor D. Choice of technique in surgery for congenital cataract. Trans Ophthalmol Soc UK 1981; 101 : 114-7.

- Dudgeon J A, Peckham C S, Marshall W C, Smithells R W, Sheppard S. National congenital rubella surveillance programme. Health Trends 1973 ; 5: 75-9.

- Taylor D, Vaegan, Morris J A, Rogers J E, Warland J. Amblyopia in bilateral infantile and juvenile cataract. Trans Ophthalmol Soc UK 1979; 99: 170-5.

8 Morris J, Taylor D, Rogers J E, Vaegan, Warland J. Contact lens treatment of aphakic infants and children. J Br Contact Lens Assoc 1979; 2: 22-30.

9 Beller R, Hoyt C S, Marg E, Odom J V. Good visual function after neonatal surgery for congenital monocular cataracts. Am J Ophthalmol $1981 ; 91$ : 559-65.

10 Tommila V, Tarkkanen A. Incidence of loss of vision in the healthy eye in amblyopia. Br J Ophthalmol 1981; 65: 575-7.

11 Frank K E, Purnell E W. Subretinal neovascularization following rubella retinopathy. Am J Ophthalmol 1978; 86: 462-6.

DAVID TAYLOR Hospital for Sick Children, Great Ormond Street, London WC1N $3 J H$

NOEL S C Rice Moorfields Eye Hospital, City Road, London ECIW $2 P D$

Reprint requests to $\mathrm{D}$ Taylor. 